\title{
Stability analysis by fixed point theorems for a class of non-linear Caputo nabla fractional difference equation
}

\author{
Rabia Ilyas Butt' ${ }^{1}$, Thabet Abdeljawad ${ }^{2,3,4^{*}}$ (D) and Mujeeb ur Rehman'
}

\section{"Correspondence:}

tabdeljawad@psu.edu.sa

${ }^{2}$ Department of Mathematics and

General Sciences, Prince Sultan

University, Riyadh, Saudi Arabia

${ }^{3}$ Department of Medical Research,

China Medical University, Taichung,

Taiwan

Full list of author information is

available at the end of the article

\begin{abstract}
Fractional difference equations have become important due to their qualitative properties and applications in discrete modeling. Stability analysis of solutions is one of the most widely used qualitative properties with tremendous applications. In this paper, we investigate the existence and stability results for a class of non-linear Caputo nabla fractional difference equations. To obtain the existence and stability results, we use Schauder's fixed point theorem, the Banach contraction principle and Krasnoselskii's fixed point theorem. The analysis of the theoretical results depends on the structure of nabla discrete Mittag-Leffler functions. An example is provided to illustrate the theoretical results.
\end{abstract}

Keywords: Caputo nabla fractional difference; Stability; Schauder's fixed point theorem; Banach contraction principle; Krasnoselskii's fixed point theorem

\section{Introduction}

The advancement in the theory of the fractional calculus is more than three centuries old evolving along with the classical integer order calculus. Due to the superior merits of memory and hereditary properties, fractional calculus is getting more attention among researchers [1,2]. Nowadays, fractional derivatives have been extensively used to model mechanical and electrical properties of assorted real materials. Furthermore, fractional derivatives have also been broadly used into different types of physical, chemical and biological phenomena [3-5]. Discrete fractional calculus has generated interest in recent years (see [6] and the references therein). For the trend of fractional calculus with different types of discrete exponential and Mittag-Leffler kernels we attract the attention of the reader to the recent manuscripts $[7,8]$ on kernels. This trend took place after the publication of continuous counterparts $[9,10]$. Specially, fractional difference equations have become a popular topic recently. Using different approaches existence, uniqueness and then analysis of attractivity, stability and asymptotic stability of the solutions of fractional difference equations are most important topics in this direction [11-15].

Fixed point theorems are widely used for existence and uniqueness purposes. Furthermore, they are used to investigate the attractivity of solutions as well as stability and the

(c) The Author(s) 2020. This article is licensed under a Creative Commons Attribution 4.0 International License, which permits use sharing, adaptation, distribution and reproduction in any medium or format, as long as you give appropriate credit to the original author(s) and the source, provide a link to the Creative Commons licence, and indicate if changes were made. The images or other third party material in this article are included in the article's Creative Commons licence, unless indicated otherwise in a credit line to the material. If material is not included in the article's Creative Commons licence and your intended use is not permitted by statutory regulation or exceeds the permitted use, you will need to obtain permission directly from the copyright holder. To view a copy of this licence, visit http://creativecommons.org/licenses/by/4.0/. 
asymptotic stability. In 2017, Abdeljawad et al. [16] used the Krasnoselskii fixed point theorem for a Caputo q-difference equation to investigate the existence of a solution. In 2018, Zhang and Zhou investigated existence and attractivity of solutions of RiemannLiouville-like fractional difference equations using the Picard iteration method and then they used Schauder's fixed point theorem for the required results [17]. Furthermore, they also proved results using weighted space. In 2018, Ardjouni et al. [18] used the Krasnoselskii fixed point theorem for a stability analysis of a nabla fractional difference equation. In 2018, Arjumand worked on existence and stability analysis of a non-linear Caputo fractional differential equation using the Krasnoselskii fixed point theorem by considering the solution of a fractional differential equation [19]. Besides, for help on stability refer to [20-25] and the references therein. Motivated by all the above-mentioned papers, here we consider the following fractional difference equation with initial condition:

$$
{ }^{c} \nabla_{0}^{v} x(t)=\lambda x(t)+\mathscr{F}(t, x(t)), \quad x(0)=x_{0}, t \in \mathbb{N}_{0} .
$$

Here ${ }^{c} \nabla_{0}^{v}$ represents the Caputo nabla fractional difference operator with $0<v \leq 1$ and a continuous function $\mathscr{F}$ is defined as $\mathscr{F}: \mathbb{N}_{0} \times \mathbb{R} \rightarrow \mathbb{R}$ with $\mathscr{F}(t, 0)=0$. Here we use the approach as mentioned in [19] by considering the solution of Eq. (1) in terms of the nabla discrete Mittag-Leffler function and then using its properties, we discuss the existence of solutions and then attractivity, stability and finally asymptotic stability of solutions of the above-mentioned problem. To the best of our knowledge, this approach to studying the stability using the fixed point theorem has never been followed by any researcher.

We arranged our current paper as follows: Sect. 2 contains some basic definitions, notations, lemmas and graphic analysis for the nabla discrete Mittag-Leffler functions. Section 3 intends to investigate the existence and stability results. Finally an example is provided with concluding remarks.

\section{Preliminaries and essential tools}

In this section, we present some basic nabla notations, definitions $[6,26,27]$ and lemmas that are helpful in proving our main results. The graphs of the nabla discrete Mittag-Leffler functions will be shown and the behavior at infinity will be investigated as well.

Definition 1 For $x: \mathbb{N}_{a+1} \rightarrow \mathbb{R}$ and $v>0$, the nabla fractional sum is defined as

$$
\nabla_{a}^{-v} x(t)=\frac{1}{\Gamma(\nu)} \sum_{\tau=a+1}^{t}(t-\rho(\tau))^{\overline{\nu-1}} x(\tau), \quad t \in \mathbb{N}_{a},
$$

where $\rho(\tau)=\tau-1$. Meanwhile, $\nabla_{a}^{-0}$ is taken as the identity operator.

Definition 2 For $x: \mathbb{N}_{a} \rightarrow \mathbb{R}, v \in \mathbb{R}^{+}$and choose a natural number $n$ such that $n-1<v<$ $n$, then the $\nu$ th order nabla fractional difference is defined as

$$
\nabla_{a}^{v} x(t)=\frac{1}{\Gamma(-v)} \sum_{\tau=a+1}^{t}(t-\rho(\tau))^{\overline{-\nu-1}} x(\tau), \quad t \in \mathbb{N}_{a+1},
$$

where $\nabla_{a}^{0}$ is taken as the identity operator. 
Definition 3 For $x: \mathbb{N}_{a-n+1} \rightarrow \mathbb{R}$ and $v>0$. The $v$ th order Caputo nabla fractional difference of $x$ is given by

$$
{ }^{c} \nabla_{a}^{v} x(t)=\nabla_{a}^{-(n-v)}\left[\nabla^{n} x(t)\right], \quad t \in \mathbb{N}_{a+1}, n=\lceil\nu\rceil .
$$

Here ${ }^{c} \nabla_{a}^{0}$ is taken as the identity operator.

Definition 4 ([28]) Any subset of sequences in $l_{0}^{\infty}$ is called uniformly Cauchy (or equi Cauchy) if for every $\epsilon>0$, we have an integer $N$ such that, for any sequence $x=\{x(n)\}$ and $i, j>N$, we must have $|x(i)-x(j)|<\epsilon$.

Theorem 1 ([28], Discrete Arzela-Ascoli's theorem) Any subset of $l_{0}^{\infty}$ which is bounded and uniformly Cauchy is called relatively compact.

Theorem 2 ([17], Schauder's fixed point theorem) Let $\mathscr{N}$ be a non-empty, closed and convex subset of a Banach space $S$. Further assume a continuous mapping $\mathscr{T}: \mathscr{N} \rightarrow \mathscr{N}$ such that $\mathscr{T} \mathscr{N}$ is a relatively compact subset of $S$. Then $\mathscr{T}$ admits a unique fixed point in $\mathscr{N}$. That is, $\mathscr{T} x=x$ for $x \in \mathscr{N}$.

Theorem 3 (Banach contraction principle) Let $\mathscr{N}$ be a non-empty complete metric space with a contraction mapping $\mathscr{T}: \mathscr{N} \rightarrow \mathscr{N}$. Then $\mathscr{T}$ has at least one fixed point in $\mathscr{N}$. That is, there exists $x \in \mathscr{N}$ such that $\mathscr{T} x=x$.

Now we state Krasnoselskii's fixed point theorem.

Theorem 4 ([29], Krasnoselskii fixed point theorem) Let $\mathscr{N}$ be a non-empty, closed and convex subset of a Banach space $(S,\|\cdot\|)$. Suppose that $\mathscr{T}_{1}$ and $\mathscr{T}_{2}$ maps $\mathscr{N}$ into $S$ such that

(i) $\mathscr{T}_{1} x+\mathscr{T}_{2} y \in \mathscr{N}$ for all $x, y \in \mathscr{N}$,

(ii) $\mathscr{T}_{1}$ is continuous and $\mathscr{T}_{1} \mathscr{N}$ is contained in a compact set of $S$,

(iii) $\mathscr{T}_{2}$ is a contraction.

Then $\mathscr{T}$ admits a fixed point in $\mathscr{N}$ such that $\mathscr{T}_{1} x+\mathscr{T}_{2} x=x$.

Lemma 1 ([27]) Let $0<v \leq 1, a \in \mathbb{R}$ and consider the nabla Caputo nonhomogeneous fractional difference equation

$$
{ }^{c} \nabla_{a}^{v} z(t)=\lambda z(t)+\mathscr{F}(t), \quad z(a)=a_{0}, t \in \mathbb{N}_{a},
$$

then its solution is given by

$$
z(t)=a_{0} E_{\bar{v}}(\lambda, t-a)+\sum_{\tau=a+1}^{t} E_{\overline{\bar{v}, \nu}}(\lambda, t-\rho(\tau)) \mathscr{F}(\tau) .
$$

Remark 1 Following the above lemma, the solution of problem (1) is given by

$$
x(t)=x_{0} E_{\bar{v}}(\lambda, t)+\sum_{\tau=1}^{t} E_{\overline{\nu, v}}(\lambda, t-\rho(\tau)) \mathscr{F}(\tau, x(\tau)) .
$$


Definition 5 ([27]) For $\lambda \in \mathbb{R},|\lambda|<1$ and $\nu, \beta, t \in \mathbb{C}$ with $\Re(\nu)>0$, the nabla discrete Mittag-Leffler functions are defined as follows:

$$
E_{\overline{v, \beta}}(\lambda, t)=\sum_{m=0}^{\infty} \lambda^{m} \frac{t^{\overline{m v+\beta-1}}}{\Gamma(m v+\beta)} .
$$

For $\beta=1$, the one parameter nabla discrete Mittag-Leffler function can be written as

$$
E_{\bar{v}}(\lambda, t)=\sum_{m=0}^{\infty} \lambda^{m} \frac{t^{\overline{m v}}}{\Gamma(m v+1)} .
$$

Remark 2 (The graphs and behavior of nabla discrete Mittag-Leffler functions at $\infty$ ) Motivated by Remark 1 in [30] and for the sake of benefiting in the proof of our main results, the numerical evidence, as illustrated in Fig. 1 and Fig. 2, shows that, for $t \in \mathbb{R}^{+}$, $0<v \leq 1$, the one and two parameter nabla discrete Mittag-Leffler functions are decreasing functions of $t$ and are bounded above by 1 . That is, $E_{\bar{\nu}}(\lambda, t) \leq 1$ and $E_{\overline{v, v}}(\lambda, t) \leq 1$, where $-1<\lambda<0$ and $-v<\lambda<0$. Moreover, it is to be noted that $\lim _{t \rightarrow \infty} E_{\bar{v}}(\lambda, t)=0$ and $\lim _{t \rightarrow \infty} E_{\overline{v, v}}(\lambda, t)=0$.

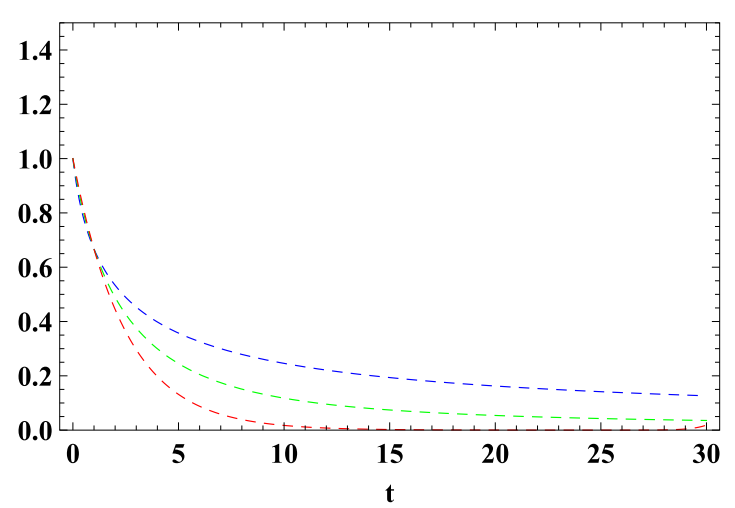

Figure $1 E_{\bar{v}}(\lambda, t)$ for $\lambda=-0.5, v=0.6,0.8,1$

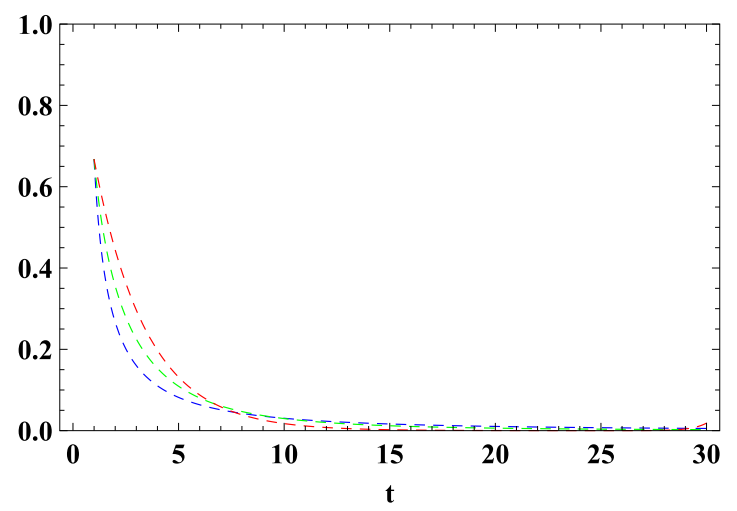

Figure $2 E_{\overline{v, v}}(\lambda, t)$ for $\lambda=-0.5, v=0.6,0.8,1$ 
Definition 6 ([19]) $x=\varphi(t)$ being a solution of Eq. (1) is called:

(i) stable, if for every $\epsilon>0$ and $t_{0} \geq 0$, there exists a $\delta>0$ depending on $t_{0}$ and $\epsilon$ such that, for $\left|x_{0}-\varphi\left(t_{0}\right)\right| \leq \delta\left(t_{0}, \epsilon\right)$, we have, for all $t \geq t_{0},\left|x\left(t, x_{0}, t_{0}\right)-\varphi(t)\right|<\epsilon$;

(ii) attractive, if there exists $\zeta\left(t_{0}\right)>0$ such that $\left\|x_{0}\right\| \leq \zeta$ implies $\lim _{t \rightarrow \infty} x\left(t, x_{0}, t_{0}\right)=0$;

(iii) asymptotically stable if it is attractive and stable.

\section{Main results}

In this section, we present existence and stability of the solutions of Eq. (1).

\subsection{The existence and uniqueness theorems}

Let $l_{0}^{\infty}$ be the set of all real sequences $x=\{x(t)\}_{t=0}^{\infty}$ from the starting point $t=0$. The space is endowed with the supremum norm $\|x\|=\sup _{t \in \mathbb{N}_{0}}|x(t)| . l_{0}^{\infty}$ is a Banach space.

Theorem 5 Let us consider the solution of Eq. (1) as mentioned in Eq. (4). Assume a continuous and bounded function $\mathscr{F}$, which obeys

$\left(A_{1}\right) \mathscr{F}: \mathbb{N}_{0} \times \mathbb{R} \rightarrow \mathbb{R}$ satisfies the Lipschitz condition with $\mathscr{L}>0$ being a Lipschitz constant,

$$
\|\mathscr{F}(t, x)-\mathscr{F}(t, y)\| \leq \mathscr{L}\|x-y\|, \quad \forall t \in \mathbb{N}_{0}^{b}, b>0 .
$$

Then using Schauder's fixed point theorem, there exists at least one solution of Eq. (1).

Proof Let us consider a non-empty, closed and convex subset $\mathscr{K}=\left\{x: x \in l_{0}^{\infty},\|x\| \leq \Lambda\right\}$; also assume that $\|\mathscr{F}(t, x)\| \leq \mathscr{R}, \forall(t, x) \in \mathbb{N}_{0}^{b} \times \mathbb{R}$. Furthermore, consider a mapping $\mathscr{T}$ on $\mathscr{K}$ as given by

$$
\mathscr{T} x(t)=x_{0} E_{\bar{v}}(\lambda, t)+\sum_{\tau=1}^{t} E_{\overline{\nu, v}}(\lambda, t-\rho(\tau)) \mathscr{F}(\tau, x(\tau)) .
$$

First of all we show that $\mathscr{T}$ maps $\mathscr{K}$ into $\mathscr{K}$ :

$$
\begin{aligned}
|\mathscr{T} x(t)| & =\left|x_{0} E_{\bar{v}}(\lambda, t)+\sum_{\tau=1}^{t} E_{\overline{\nu, v}}(\lambda, t-\rho(\tau)) \mathscr{F}(\tau, x(\tau))\right| \\
& \leq\left|x_{0}\right|\left|E_{\bar{v}}(\lambda, t)\right|+\int_{0}^{t}\left|E_{\overline{v, v}}(\lambda, t-\rho(\tau))\right||\mathscr{F}(\tau, x(\tau))| \nabla \tau \\
& \leq\left|x_{0}\right|+\mathscr{R} b \leq \Lambda .
\end{aligned}
$$

Now we have to show that $\mathscr{T}$ is relatively compact, for this purpose consider $0 \leq t_{1} \leq t_{2} \leq$ $b$ so we get

$$
\begin{aligned}
& \left|\mathscr{T} x\left(t_{2}\right)-\mathscr{T} x\left(t_{1}\right)\right| \\
& \quad \leq\left|x_{0}\left(E_{\bar{v}}\left(\lambda, t_{2}\right)-E_{\bar{v}}\left(\lambda, t_{1}\right)\right)\right| \\
& \quad+\left|\left(\sum_{\tau=1}^{t_{2}} E_{\overline{\nu, v}}\left(\lambda, t_{2}-\rho(\tau)\right)-\sum_{\tau=1}^{t_{1}} E_{\overline{v, v}}\left(\lambda, t_{1}-\rho(\tau)\right)\right) \mathscr{F}(\tau, x(\tau))\right| \\
& \leq\left|x_{0}\left(E_{\bar{v}}\left(\lambda, t_{2}\right)-E_{\bar{v}}\left(\lambda, t_{1}\right)\right)\right|
\end{aligned}
$$




$$
\begin{aligned}
& \quad+\left|\left(\sum_{\tau=1}^{t_{1}} E_{\overline{\bar{v}, v}}\left(\lambda, t_{2}-\rho(\tau)\right)-\sum_{\tau=1}^{t_{1}} E_{\overline{v, v}}\left(\lambda, t_{1}-\rho(\tau)\right)\right) \mathscr{F}(\tau, x(\tau))\right| \\
& +\left|\sum_{\tau=t_{1}+1}^{t_{2}} E_{\overline{v, v}}\left(\lambda, t_{2}-\rho(\tau)\right) \mathscr{F}(\tau, x(\tau))\right| \\
& \leq\left|x_{0}\left(E_{\bar{v}}\left(\lambda, t_{2}\right)-E_{\bar{v}}\left(\lambda, t_{1}\right)\right)\right| \\
& +\int_{0}^{t_{1}}\left|\left(E_{\overline{v, v}}\left(\lambda, t_{2}-\rho(\tau)\right)-E_{\bar{v}, \bar{v}}\left(\lambda, t_{1}-\rho(\tau)\right)\right)\right||\mathscr{F}(\tau, x(\tau))| \nabla \tau \\
& \quad+\int_{t_{1}}^{t_{2}}\left|E_{\overline{v, v}}\left(\lambda, t_{2}-\rho(\tau)\right)\right||\mathscr{F}(\tau, x(\tau))| \nabla \tau \\
& \leq\left|x_{0}\left(E_{\bar{v}}\left(\lambda, t_{2}\right)-E_{\bar{v}}\left(\lambda, t_{1}\right)\right)\right| \\
& +\int_{0}^{t_{1}}\left|\left(E_{\overline{v, v}}\left(\lambda, t_{2}-\rho(\tau)\right)-E_{\bar{v}, \bar{v}}\left(\lambda, t_{1}-\rho(\tau)\right)\right)\right| \mathscr{R} \nabla \tau \\
& \quad+\mathscr{R}\left(t_{2}-t_{1}\right) .
\end{aligned}
$$

Hence it follows that $\left|\mathscr{T} x\left(t_{2}\right)-\mathscr{T} x\left(t_{1}\right)\right| \rightarrow 0$ as $t_{1} \rightarrow t_{2}$.

Now in order to show the continuity of $\mathscr{T}$, let us consider a sequence $x_{n}$ which converges to $x$, then we have

$$
\begin{aligned}
\left|\mathscr{T} x_{n}(t)-\mathscr{T} x(t)\right| & \leq\left|\sum_{\tau=1}^{t} E_{\overline{v, v}}(\lambda, t-\rho(\tau))\left(\mathscr{F}\left(\tau, x_{n}(\tau)\right)-\mathscr{F}(\tau, x(\tau))\right)\right| \\
& \leq \int_{0}^{t}\left|E_{\overline{v, v}}(\lambda, t-\rho(\tau))\right|\left|\mathscr{F}\left(\tau, x_{n}(\tau)\right)-\mathscr{F}(\tau, x(\tau))\right| \nabla \tau \\
& \leq \mathscr{L}\left\|x_{n}-x\right\| \int_{0}^{t} \nabla \tau \leq \mathscr{L} b\left\|x_{n}-x\right\|,
\end{aligned}
$$

we can see easily $\mathscr{T} x_{n} \rightarrow \mathscr{T} x$ as $x_{n} \rightarrow x$. Hence using Arzela-Ascoli's theorem, $\mathscr{T} \mathscr{K}$, being a bounded and uniformly Cauchy subset of $l_{0}^{\infty}$, is relatively compact.

Hence by Schauder's fixed point theorem there exists at least one fixed point of $\mathscr{T}$ in $\mathscr{K}$. Furthermore, if all functions $x$ in $\mathscr{K}$ tend to 0 as $t \rightarrow \infty$ then solutions of Eq. (1) tend to zero as $t \rightarrow \infty$ and hence are called attractive solutions.

\subsection{The stability results}

In the light of the fixed point results presented in the above subsection, we present some stability results in what follows.

Theorem 6 Assume that a bounded, continuous function $\mathscr{F}$ satisfies the following conditions,

$\left(A_{2}\right)\|\mathscr{F}(t, x)-\mathscr{F}(t, y)\| \leq \mathscr{L}(t)\|x-y\|$,

$\left(A_{3}\right) \int_{0}^{t} \mathscr{L}(\tau) \nabla \tau \rightarrow 0$ as $t \rightarrow \infty$, where $\mathscr{M}=\sup _{t \in \mathbb{N}_{0}} \int_{0}^{t} \mathscr{L}(\tau) \nabla \tau$,

then using the Banach contraction principle, there exists a unique solution of Eq. (1). Moreover, the solution is attractive. 
Proof Define a set $\mathscr{E}=\left\{x \in l_{0}^{\infty},\|x\| \leq \epsilon\right.$ and $x(t) \rightarrow 0$ as $\left.t \rightarrow \infty\right\}$. Furthermore, assume that

$$
|\mathscr{F}(t, x)| \leq|\mathscr{F}(t, x)-\mathscr{F}(t, 0)|+|\mathscr{F}(t, 0)| \leq \mathscr{L}(t)|x-0|+0=\mathscr{L}(t)\|x\| .
$$

Now we define a mapping $\mathscr{T}$ on $\mathscr{E}$ as follows:

$$
\mathscr{T} x(t)=x_{0} E_{\bar{v}}(\lambda, t)+\sum_{\tau=1}^{t} E_{\overline{\nu, v}}(\lambda, t-\rho(\tau)) \mathscr{F}(\tau, x(\tau)) .
$$

Continuity of $\mathscr{T} x$ is followed by $x \in \mathscr{E}$. First of all we prove that $\mathscr{T}$ maps $\mathscr{E}$ into itself:

$$
\begin{aligned}
|\mathscr{T} x(t)| & =\left|x_{0} E_{\bar{v}}(\lambda, t)+\sum_{\tau=1}^{t} E_{\overline{v, v}}(\lambda, t-\rho(\tau)) \mathscr{F}(\tau, x(\tau))\right| \\
& \leq\left|x_{0}\right|\left|E_{\bar{v}}(\lambda, t)\right|+\int_{0}^{t}\left|E_{\bar{v}, \bar{v}}(\lambda, t-\rho(\tau))\right||\mathscr{F}(\tau, x(\tau))| \nabla \tau \\
& \leq\left|x_{0}\right|+\int_{0}^{t} \mathscr{L}(\tau)\|x(\tau)\| \nabla \tau \\
& \leq\left|x_{0}\right|+\epsilon \int_{0}^{t} \mathscr{L}(\tau) \nabla \tau \leq\left|x_{0}\right|+\epsilon \mathscr{M} \leq \epsilon .
\end{aligned}
$$

Hence $\mathscr{T}$ maps $\mathscr{E}$ into itself. Now we show that $\mathscr{T} x(t) \rightarrow 0$ as $t \rightarrow \infty$. We can see easily in Figs. 1 and 2 that $\lim _{t \rightarrow \infty} E_{\bar{v}}(\lambda, t) \rightarrow 0$ and $\lim _{t \rightarrow \infty} E_{\overline{\nu, v}}(\lambda, t-\rho(\tau)) \rightarrow 0$. So we have $\lim _{t \rightarrow \infty} x_{0} E_{\bar{v}}(\lambda, t) \rightarrow 0$. Also

$$
\begin{aligned}
\left|\int_{0}^{t} E_{\overline{\bar{v}, v}}(\lambda, t-\rho(\tau)) \mathscr{F}(\tau, x(\tau)) \nabla \tau\right| & \leq \int_{0}^{t}\left|E_{\overline{v, v}}(\lambda, t-\rho(\tau))\right||\mathscr{F}(\tau, x(\tau))| \nabla \tau \\
& \leq \int_{0}^{t}\left|E_{\overline{\bar{v}, v}}(\lambda, t-\rho(\tau))\right| \mathscr{L}(\tau)\|x(\tau)\| \nabla \tau \\
& \leq \epsilon \int_{0}^{t} \mathscr{L}(\tau) \nabla \tau \leq \epsilon \mathscr{M} .
\end{aligned}
$$

Thus $\left|\int_{0}^{t} E_{\overline{v, v}}(\lambda, t-\rho(\tau)) \mathscr{F}(\tau, x(\tau)) \nabla \tau\right| \rightarrow 0$ as $t \rightarrow \infty$. Hence $\mathscr{T} x(t) \rightarrow 0$ as $t \rightarrow \infty$. Now we show that $\mathscr{T}$ is a contraction mapping:

$$
\begin{aligned}
\left|\mathscr{T}_{x}(t)-\mathscr{T} y(t)\right| & \leq \int_{0}^{t}\left|E_{\overline{\nu, v}}(\lambda, t-\rho(\tau))\right||\mathscr{F}(\tau, x(\tau))-\mathscr{F}(\tau, y(\tau))| \nabla \tau \\
& \leq\|x-y\| \int_{0}^{t} \mathscr{L}(\tau) \nabla \tau \leq \mathscr{M}\|x-y\| .
\end{aligned}
$$

For $\mathscr{M}<1, \mathscr{T}$ is contraction. Hence by the contraction mapping principle Eq. (1) has a unique solution and furthermore, since all functions $x$ in $\mathscr{E}$ tend to 0 as $t \rightarrow \infty$, a solution of Eq. (1) tends to zero as $t \rightarrow \infty$, and hence is called an attractive solution.

Theorem 7 Let $x$ be solution of Eq. (1) and $\hat{x}$ be a solution of Eq. (1) satisfying the initial condition $\hat{x}(0)=\hat{x}_{0}$. Moreover, let there for very small $\epsilon>0$ exist $\delta=(1-\mathscr{M}) \epsilon$. Then solutions of Eq. (1) are stable. 
Proof Since $x$ is a solution of Eq. (1) and $\hat{x}$ is also a solution of Eq. (1) satisfying the initial condition $\hat{x}(0)=\hat{x}_{0}$, we have

$$
\begin{aligned}
|x(t)-\hat{x}(t)| \leq & \left|x_{0} E_{\bar{v}}(\lambda, t)-\hat{x}_{0} E_{\bar{v}}(\lambda, t)\right| \\
& +\left|\sum_{\tau=1}^{t} E_{\overline{\nu, v}}(\lambda, t-\rho(\tau)) \mathscr{F}(\tau, x(\tau))-\sum_{\tau=1}^{t} E_{\overline{v, v}}(\lambda, t-\rho(\tau)) \mathscr{F}(\tau, \hat{x}(\tau))\right| \\
\leq & \left|x_{0}-\hat{x}_{0}\right|\left|E_{\bar{v}}(\lambda, t)\right|+\int_{0}^{t}\left|E_{\overline{\nu, v}}(\lambda, t-\rho(\tau))\right||\mathscr{F}(\tau, x(\tau))-\mathscr{F}(\tau, \hat{x}(\tau))| \nabla \tau \\
\leq & \left|x_{0}-\hat{x}_{0}\right|+\int_{0}^{t} \mathscr{L}(\tau)|x(\tau)-\hat{x}(\tau)| \nabla \tau \leq\left|x_{0}-\hat{x}_{0}\right|+\|x-\hat{x}\| \int_{0}^{t} \mathscr{L}(\tau) \nabla \tau \\
\leq & \left|x_{0}-\hat{x}_{0}\right|+\|x-\hat{x}\| \mathscr{M} .
\end{aligned}
$$

Hence we have

$$
\|x-\hat{x}\| \leq \frac{1}{1-\mathscr{M}}\left\|x_{0}-\hat{x}_{0}\right\|
$$

Then, for any $\epsilon>0$, let $\delta=(1-\mathscr{M}) \epsilon$ so for $\left\|x_{0}-\hat{x}_{0}\right\|<\delta$ we have $\|x-\hat{x}\|<\epsilon$. Therefore, the solutions of Eq. (1) are stable. This completes the proof.

Remark 3 From Theorem (6) and Theorem (7), it is clear that a solution of Eq. (1) is asymptotically stable.

Theorem 8 Assume that we have a bounded, continuous function $\mathscr{F}$ satisfying assumptions $\left(A_{2}\right)$ and $\left(A_{3}\right)$. Then using Krasnoselskii's fixed point theorem, Eq. (1) has at least one solution.

Proof Let us consider a non-empty, closed, convex subset of a Banach space $l_{0}^{\infty}$ defined as $\mathscr{N}=\left\{x: x \in l_{0}^{\infty},|x(t)| \leq m, \forall t \in \mathbb{N}_{0}\right\}$. Furthermore, define operators $\mathscr{T}_{1}$ and $\mathscr{T}_{2}$ on $\mathscr{N}$ as follows:

$$
\begin{aligned}
& \mathscr{T}_{1} x(t)=x_{0} E_{\bar{\nu}}(\lambda, t), \\
& \mathscr{T}_{2} x(t)=\sum_{\tau=1}^{t} E_{\overline{\nu, \nu}}(\lambda, t-\rho(\tau)) \mathscr{F}(\tau, x(\tau))=\int_{0}^{t} E_{\overline{\bar{v}, \nu}}(\lambda, t-\rho(\tau)) \mathscr{F}(\tau, x(\tau)) \nabla \tau .
\end{aligned}
$$

As is well known, $x$ being fixed point of the operator $\mathscr{T}_{x}=\mathscr{T}_{1} x+\mathscr{T}_{2} x$ is a solution of Eq. (1). Following the three steps as mentioned in Theorem 4, we present our proof as follows. In the first step, we prove that $\mathscr{T}$ maps $\mathscr{N}$ into $\mathscr{N}$ i.e. for any $x, y \in \mathscr{N}$, we have to show that $\mathscr{T}_{1} x(t)+\mathscr{T}_{2} y(t) \in \mathscr{N}$ :

$$
\begin{aligned}
\left|\mathscr{T}_{1} x(t)+\mathscr{T}_{2} y(t)\right| & =\left|x_{0} E_{\bar{v}}(\lambda, t)+\sum_{\tau=1}^{t} E_{\overline{\nu, v}}(\lambda, t-\rho(\tau)) \mathscr{F}(\tau, y(\tau))\right| \\
& \leq\left|x_{0} E_{\bar{v}}(\lambda, t)\right|+\left|\sum_{\tau=1}^{t} E_{\overline{v, v}}(\lambda, t-\rho(\tau)) \mathscr{F}(\tau, y(\tau))\right|
\end{aligned}
$$




$$
\begin{aligned}
& \leq\left|x_{0}\right|+\int_{0}^{t}\left|E_{\overline{\nu, v}}(\lambda, t-\rho(\tau))\right||\mathscr{F}(\tau, y(\tau))| \nabla \tau \\
& \leq\left|x_{0}\right|+\int_{0}^{t} \mathscr{L}(\tau)|y(\tau)| \nabla \tau \\
& \leq\left|x_{0}\right|+\mathscr{M} m \leq m .
\end{aligned}
$$

Hence $\mathscr{T} \mathscr{N} \subset \mathscr{N}$. In the second step, in order to prove that $\mathscr{T}_{1}$ is continuous, let us consider a sequence $x_{n}$ such that $x_{n} \rightarrow x$.

$$
\left|\mathscr{T}_{1} x_{n}(t)-\mathscr{T}_{1} x(t)\right|=\left|x_{0} E_{\bar{v}}(\lambda, t)-x_{0} E_{\bar{v}}(\lambda, t)\right|=0,
$$

so for $x_{n} \rightarrow x, \mathscr{T}_{1} x_{n} \rightarrow \mathscr{T}_{1} x$. Hence $\mathscr{T}_{1}$ is continuous. Now we show that $\mathscr{T}_{1}(\mathscr{N})$ resides in a relatively compact set of $l_{0}^{\infty}$. Taking $t_{1} \leq t_{2} \leq H$, we have

$$
\begin{aligned}
\left|\mathscr{T}_{1} x\left(t_{2}\right)-\mathscr{T}_{1} x\left(t_{1}\right)\right| & =\left|x_{0} E_{\bar{\nu}}\left(\lambda, t_{2}\right)-x_{0} E_{\bar{\nu}}\left(\lambda, t_{1}\right)\right| \\
& =\left|x_{0}\right|\left|E_{\bar{\nu}}\left(\lambda, t_{2}\right)-E_{\bar{\nu}}\left(\lambda, t_{1}\right)\right|
\end{aligned}
$$

as $t_{1} \rightarrow t_{2}$, we get $\left|\mathscr{T}_{1} x\left(t_{2}\right)-\mathscr{T}_{1} x\left(t_{1}\right)\right| \rightarrow 0$. Hence $\mathscr{T}_{1}(\mathscr{N})$ resides in a relatively compact set of $l_{0}^{\infty}$.

In the last step, we show that $\mathscr{T}_{2}$ is contraction. Letting $x, y \in \mathscr{N}$, we have

$$
\begin{aligned}
& \left|\mathscr{T}_{2} x(t)-\mathscr{T}_{2} y(t)\right| \\
& \quad=\left|\sum_{\tau=1}^{t} E_{\overline{, v}}(\lambda, t-\rho(\tau)) \mathscr{F}(\tau, x(\tau))-\sum_{\tau=1}^{t} E_{\overline{\nu, v}}(\lambda, t-\rho(\tau)) \mathscr{F}(\tau, y(\tau))\right| \\
& \quad=\left|\int_{0}^{t} E_{\overline{\nu, v}}(\lambda, t-\rho(\tau)) \mathscr{F}(\tau, x(\tau)) \nabla \tau-\int_{0}^{t} E_{\overline{v, v}}(\lambda, t-\rho(\tau)) \mathscr{F}(\tau, y(\tau)) \nabla \tau\right| \\
& \quad \leq \int_{0}^{t}\left|E_{\overline{\nu, v}}(\lambda, t-\rho(\tau))\right||(\mathscr{F}(\tau, x(\tau))-\mathscr{F}(\tau, y(\tau)))| \nabla \tau \\
& \quad \leq \int_{0}^{t} \mathscr{L}(\tau)|x(\tau)-y(\tau)| \nabla \tau \\
& \quad \leq\|x-y\| \int_{0}^{t} \mathscr{L}(\tau) \nabla \tau \leq \mathscr{M}\|x-y\| .
\end{aligned}
$$

For $\mathscr{M}<1, \mathscr{T}_{2}$ is contraction.

Hence according to Theorem $4, \mathscr{T}$ has a fixed point in $\mathscr{N}$ which is a solution of Eq. (1).

Remark 4 According to Theorem (8), the solutions of Eq. (1) exist and are in $\mathscr{N}$. Furthermore, if all functions $x$ in $\mathscr{N}$ tend to 0 as $t \rightarrow \infty$, then the solutions of Eq. (1) are attractive.

Remark 5 From Theorem 7 and Theorem 8, it is clear that the solution of Eq. (1) is asymptotically stable. 


\section{Example}

Consider the fractional difference equation as follows:

$$
{ }^{c} \nabla_{0}^{0.6} x(t)=-0.5 x(t)+t^{-1.7} \sin x(t), \quad x(0)=x_{0}, t \in \mathbb{N}_{1},
$$

where $\mathscr{F}(t, x(t))=t^{-\overline{1.7}} \sin x(t), t \in \mathbb{N}_{1}$.

According to Lemma 1, a solution of the problem under consideration can be written as

$$
x(t)=x_{0} E_{\overline{0.6}}(-0.5, t)+\sum_{\tau=1}^{t} E_{\overline{0.6,0.6}}(-0.5, t-\rho(\tau)) \tau^{\overline{-1.7}} \sin x(\tau) .
$$

We can see easily that the function $\mathscr{F}(t, x(t))$ satisfies condition $\left(A_{1}\right)$, so by Theorem 5 , there exists at least one solution of problem (5).

Moreover,

$$
\begin{aligned}
\|\mathscr{F}(t, x)-\mathscr{F}(t, y)\| & =\left\|t^{\overline{-1.7}} \sin x-t^{\overline{-1.7}} \sin y\right\| \\
& \leq \mathscr{L}(t)\|x-y\|,
\end{aligned}
$$

where $\mathscr{L}(t)=t^{\overline{-1.7}}$. Calculation shows that $\mathscr{M}=\sup _{t \in \mathbb{N}_{1}} \int_{0}^{t} \mathscr{L}(\tau) \nabla \tau<1$ and $\int_{0}^{t} \mathscr{L}(\tau) \nabla \tau \rightarrow$ 0 as $t \rightarrow \infty$. Hence conditions $\left(A_{2}\right)$ and $\left(A_{3}\right)$ are satisfied. So by Theorems 6 and 8 , there exists at least one solution of problem (5). Moreover, by Theorem 7 the solution is stable. Furthermore, the solution $x$ of problem (5) tends to 0 as $t \rightarrow \infty$. Hence the solution is asymptotically stable.

\section{Conclusion}

Stability analysis is one of the most important topics due to its real-life applications in controlling systems. Fractional differential as well as fractional difference equations are widely used in real-life models, fixed point theorems are essential tools for stability analysis. In this paper, we have used Schauder's fixed point theorem, the Banach contraction principle and Krasnoselskii's fixed point theorem to study the existence and stability. The analysis of the results heavily depends on the nabla discrete Mittag-Leffler functions.

Acknowledgements

The second author would like to thank Prince Sultan University for funding this work through research group Nonlinear Analysis Methods in Applied Mathematics (NAMAM) group number RG-DES-2017-01-17.

Funding

Not applicable.

Availability of data and materials

Not applicable.

Competing interests

The authors declare that they have no competing interests.

Consent for publication

Not applicable.

Authors' contributions

All authors read and approved the final manuscript. 


\section{Author details}

'Department of Mathematics, School of Natural Sciences, National University of Sciences and Technology, Islamabad, Pakistan. ${ }^{2}$ Department of Mathematics and General Sciences, Prince Sultan University, Riyadh, Saudi Arabia. ${ }^{3}$ Department of Medical Research, China Medical University, Taichung, Taiwan. ${ }^{4}$ Department of Computer Science and Information Engineering, Asia University, Taichung, Taiwan.

\section{Publisher's Note}

Springer Nature remains neutral with regard to jurisdictional claims in published maps and institutional affiliations.

Received: 9 March 2020 Accepted: 30 April 2020 Published online: 13 May 2020

\section{References}

1. Podlubny, l.: Fractional Differential Equations. Mathematics in Science and Engineering, vol. 198. Academic Press, San Diego (1999)

2. Diethelm, K.: The Analysis of Fractional Differential Equations. Lecture Notes in Mathematics, vol. 2004. Springer, Berlin (2010)

3. Hilfer, R.: Applications of Fractional Calculus in Physics. World Scientific, River Edge (2000)

4. Oldham, K.B.: Fractional differential equations in electrochemistry. Adv. Eng. Softw. 41, 9-12 (2010)

5. Magin, R.L.: Fractional calculus models of complex dynamics in biological tissues. Comput. Math. Appl. 59, 1586-1593 (2010)

6. Goodrich, C., Peterson, A.C.: Discrete Fractional Calculus. Springer, Cham (2015)

7. Abdeljawad, T.: Different type kernel h-fractional differences and their fractional $h$-sums. Chaos Solitons Fractals 116 , $146-156(2018)$

8. Abdeljawad, T.: Fractional difference operators with discrete generalized Mittag-Leffler kernels. Chaos Solitons Fractals 126, 315-324 (2019)

9. Caputo, M., Fabrizio, M.: A new definition of fractional derivative without singular kernal. Prog. Fract. Differ. Appl. 2, 73-85 (2015)

10. Atangana, A., Baleanu, D.: New fractional derivative with non-local and non-singular kernel. Therm. Sci. 20, 757-763 (2016)

11. Jarad, F., Abdeljawad, T., Baleanu, D., Biçen, K.: On the stability of some discerete fractional nonautonomous systems. Abstr. Appl. Anal. 2012, Article ID 476581, 1-9 (2012)

12. Chen, F.: Fixed points and asymptotic stability of nonlinear fractional difference equations. Acta Vet. Scand. 2011 $1-18(2011)$

13. Chen, F., Liu, Z.G.: Asymptotic stability results for nonlinear fractional difference equations. J. Appl. Math. 2012, Article ID 879657 (2012)

14. Wu, G.C., Baleanu, D.: Stability analysis of impulsive fractional difference equations. Fract. Calc. Appl. Anal. 21, 354-375 (2018)

15. Wu, G.C., Baleanu, D., Huang, L.L.: Novel Mittag-Leffler stability of linear fractional delay difference equations impulse. Appl. Math. Lett. 82, 71-78 (2018)

16. Abdeljawad, T., Alzabut, J., Zhou, H.: A Krasnoselskii existence result for nonlinear delay Caputo q-fractional difference equations with applications to Lotka-Volterra competition model. Appl. Math. E-Notes 17, 307-318 (2017)

17. Zhang, L., Zhou, Y.: Existence and attractivity of solutions for fractional difference equations. Adv. Differ. Equ. 2018 191, 1-15 (2018)

18. Ardjouni, A., Boulares, H., Djoudi, A.: Stability of nonlinear neutral nabla fractional difference equations. Commun. Optim. Theory 2018, 1-10 (2018)

19. Seemab, A., Rehman, M.: Existence and stability analysis by fixed point theorems for a class of non-linear Caputo fractional differential equations. Dyn. Syst. Appl. 27, 445-456 (2018)

20. Ali, A., Samet, B., Shah, K., Khan, R.A.: Existence and stability of solution to a toppled systems of differential equations of non-integer order. Bound. Value Probl. 2017, 16, 1-13 (2017)

21. Kumama, P., Ali, A., Shah, K., Khan, R.A.: Existence results and Hyers-Ulam stability to a class of nonlinear arbitrary order differential equations. J. Nonlinear Sci. Appl. 10, 2986-2997 (2017)

22. Ali, A., Rabieib, F., Shah, K.: On Ulam's type stability for a class of impulsive fractional differential equations with nonlinear integral boundary conditions. J. Nonlinear Sci. Appl. 10, 4760-4775 (2017)

23. Wang, J., Shah, K., Ali, A.: Existence and Hyers-Ulam stability of fractional nonlinear impulsive switched coupled evolution equations. Math. Methods Appl. Sci. 41, 2392-2402 (2018)

24. Shah, K., Wang, J., Khalil, H., Khan, R.A.: Existence and numerical solutions of a coupled system of integral BVP for fractional differential equations. Adv. Differ. Equ. 2018, 149, 1-21 (2018)

25. Khan, H., Khan, A., Chen, W., Shah, K.: Stability analysis and a numerical scheme for fractional Klein-Gordon equations Math. Methods Appl. Sci. 42, 723-732 (2019)

26. Abdeljawad, T.: On Riemann and Caputo fractional differences. Comput. Math. Appl. 62, 1602-1611 (2011)

27. Abdeljawad, T.: On delta and nabla Caputo fractional differences and dual identities. Discrete Dyn. Nat. Soc. 2013, Article ID 406910 (2013)

28. Royden, H.L. Fitzpatrick, P.M. Real Analysis. China Machine Press (2009)

29. Burton, T.A.: A fixed-point theorem of Krasnoselskii. Appl. Math. Lett. 11, 85-88 (1998)

30. Abdeljawad, T., Baleanu, D.: Monotonicity analysis of a nabla discrete fractional operator with discrete Mittag-Leffler kernel. Chaos Solitons Fractals 102, 106-110 (2017) 